syndrome, diagnosed by Dr Edward Rabe, now of Maine, USA, had failed to respond to trials of acyclovir, thiamine, carnitine, phenobarbital and diazepam, but showed immediate improvement when biotin was introduced in megadoses, $10-40 \mathrm{x}$ greater than those required in biotin deficiency states. Characteristic signs of the infantile type of biotin encephalopathy, including skin rash, alopecia, ketoacidosis, and organic acidemia, were absent, and presumably, the smaller biotin doses (5-10 mg daily) employed in cases of biotin deficiency were found ineffective. A defect in biotin transport across the blood-brain barrier was postulated, since assays of biotinidase and carboxylase were normal. See Progress in Pediatric Neurology I, II, \& III, (PNB Publishers) for reports of neonatal and infantile biotin encephalopathy. This apparent nonspecific effect of biotin in cases of idiopathic familial progressive encephalopathy might prompt trials of megadose biotin in children with chronic refractory encephalopathies.

\title{
PYRIDOXINE-DEPENDENT SEIZURES AND MRI CHANGES
}

Longitudinal brain MRI findings in two patients presenting with neonatal, pyridoxine-dependent seizures and followed for 5 to 9 years are reported from the University of California, Davis, CA. Each patient had three serial MRIs, showing progressive dilation of ventricles and atrophy of cortex and white matter. Patient 1 with AED-resistant seizures was diagnosed at 3.5 months by IV $100 \mathrm{mg}$ pyridoxine administration during EEG monitoring. Seizures characterized by lip smacking, tonic eye deviation, and limb jerking began 2 hours after birth and were initially responsive to phenobarbital. Treatment with $25 \mathrm{mg}$ oral pyridoxine daily controlled seizures, but noncompliance resulted in prolonged seizures and severe developmental disability with hypotonia at 5 year follow-up. Patient 2 developed seizures consisting of eye deviation and facial twitching and responsive to phenobarbital at 4 days after birth. They recurred occasionally up to 8 months of age, when seizures became refractory to anticonvulsants and were found to respond to pyridoxine. During follow-up for 9 years, minor motor seizures, partial and generalized, occurred only during intercurrent illness or noncompliance with pyridoxine, $25 \mathrm{mg}$ daily, treatment. Seizures were preceded by sleepiness and irritability. (Gospe SM Jr, Hecht ST. Longitudinal MRI findings in pyridoxinedependent seizures. Neurology July 1998;51:74-78). (Reprints: Dr Sidney M Gospe Jr, Child Neurology, UC Davis Medical Center, 2315 Stockton Blvd, Sacramento, CA 95817).

COMMENT. Pyridoxine-dependent seizures of neonatal onset and due to an inborn abnormality of GABA synthesis, if undiagnosed and untreated, can result in progressive cerebral atrophy and severe impairments of psychomotor development. Previous reports have emphasized that the control of seizures alone may not suffice in treating pyridoxine dependency. In order to prevent mental retardation and motor and language delay, it may be necessary to increase the dose of pyridoxine to $10 \mathrm{mg} / \mathrm{kg} /$ daily to normalize neurotoxic glutamate levels. Language and cognitive disabilities secondary to pyridoxine-dependency may be partially reversed using optimal pyridoxine dosage. (See Progress in Pediatric Neurology III, PNB Publ, 1997;pp93-98).

\section{GENE LOCATION FOR MOLYBDENUM COFACTOR DEFICIENCY}

Linkage of a molybdenum cofactor deficiency (MoCoD) gene to an 8-cM region on chromosome $6 \mathrm{p} 21.3$ has been localized by homozygosity mapping in 2 consanguineous affected kindreds of Israeli-Arab origin, including 5 patients, at the Department of Genetics, Tamkin Research Facility, Technion-Israel Institute of Technology, Haifa, Israel. These findings allow prenatal diagnosis with microsatellite markers and carrier detection of this fatal disorder. (Shalata A, 